\title{
Pancreaticopleural fistula: Report of two cases and review of literature
}

\author{
Piyush Ranjan, Rinkesh Bansal, Munish Sachdeva, Mandhir Kumar \\ Department of Gastroenterology, Sir Ganga Ram Hospital, New Delhi, India
}

\begin{abstract}
Key words

Pancreaticopleural fistula is a rare clinical problem. It is seen rarely in acute and chronic pancreatitis or after pancreatic duct trauma. It poses a diagnostic challenge. It may be silent or can present with predominant chest or abdominal symptoms. The diagnosis should be suspected if a patient presents with pleural effusion in a setting of pancreatitis or alcohol intake. The significantly raised amylase in the pleural fluid offers an important clue to the diagnosis. Computed tomography is the initial imaging of choice, which defines the pancreatic as well as chest abnormalities. The therapeutic options include medical, endoscopic, as well as surgical interventions. Although, there is no data comparing the endoscopic and surgical interventions, patients are generally treated with medical and endoscopic therapies. Surgery is reserved for those who fail medical and endoscopic therapies. Here we report two cases of pancreaticopleural fistulas that were treated successfully by endoscopic retrograde cholangiopancreatography and placement of a plastic stent in the main pancreatic duct.

Endoscopic retrograde cholangiopancreatography, pancreaticopleural fistula, stenting
\end{abstract}

\section{Introduction}

Pancreaticopleural fistula (PPF) is a type of internal fistula, wherein the pancreatic secretions drain directly into the pleural cavity. It can occur as a complication of acute and chronic pancreatitis or after a traumatic disruption of the pancreatic duct. ${ }^{[1]}$ Its prevalence is estimated to be $0.4 \%$ in patients with pancreatitis. It accounts for less than $1 \%$ of all cases of pleural effusion. ${ }^{[2]}$ It is seen more commonly in patients with chronic pancreatitis than acute pancreatitis. ${ }^{[3]}$ Therapeutic options for PPF are limited. Endoscopic retrograde cholangiopancreatography (ERCP) plays a role if a discrete leak is demonstrable.

We report two cases of PPF, which were successfully treated with ERCP and placement of a plastic stent in the main pancreatic duct.

\begin{tabular}{|l|c|}
\hline \multicolumn{2}{|c|}{ Access this article online } \\
\hline \multirow{2}{*}{$\begin{array}{l}\text { Website: } \\
\text { www.jdeonline.in }\end{array}$} & Quick Response Code \\
\hline DOI: & 10.4103/0976-5042.132425 \\
& \\
\hline
\end{tabular}

\section{Case Report}

\section{Case 1}

A 53-year-old male, with a previous diagnosis of alcohol-related chronic pancreatitis since the last five years, presented to us with a sudden onset of progressive shortness of breath. He had a previous history of a pancreatic pseudoaneurysm, which was embolized. He also had a pancreatic pseudocyst, which was not treated because he was not symptomatic.

Investigations revealed a hemoglobin of $6.1 \mathrm{~g} / \mathrm{dl}$, serum amylase $341 \mathrm{IU} / \mathrm{L}$ (reference range: 5-100 IU/L), with normal renal and liver function tests. A chest $\mathrm{x}$-ray showed a left opaque hemothorax, suggestive of pleural collection. Computed tomography (CT) angiography of the chest and abdomen showed a massive left pleural effusion and chronic calcific pancreatitis, but there was no evidence of any pseudoaneurysm [Figure 1]. Pleurocentesis was performed and one liter of hemorrhagic fluid was removed. A biochemical examination of the aspirated fluid revealed protein $3.9 \mathrm{~g} / \mathrm{dl}$ and amylase $11705 \mathrm{IU} / \mathrm{L}$. A provisional diagnosis of PPF was made and ERCP was planned. The patient had recurrence of respiratory distress and a chest $\mathrm{x}$-ray showed refilling of the pleural effusion that necessitated insertion of an intercostal chest tube. About 2.8 liters of fluid was drained in 24 hours, 
which resulted in symptomatic improvement and adequate expansion of the lung parenchyma. An ERCP pancreatogram showed that the main pancreatic duct was dilated, without any stricture or stone and leak of contrast from the distal body. A 7 F stent was placed in the pancreatic duct [Figure 2]. Chest drainage decreased to $200 \mathrm{ml}$ in the next 24 hours and became nil after two days. The chest tube was removed after four days, after confirming lung expansion on a chest x-ray [Figure 3]. There was no reaccumulation of pleural effusion in the three-month follow-up.

\section{Case 2}

A 61-year-old male presented with pain in the abdomen and was found to have cholelithiasis and a cystic mass in the region of the tail of the pancreas. About $50 \mathrm{ml}$ of fluid was aspirated under endoultrasound guidance. The analysis of fluid revealed amylase of $60668 \mathrm{IU} / \mathrm{L}$. He remained asymptomatic for three to four months and again presented with pain in the abdomen and a repeat workup showed a cystic collection in the tail of the pancreas and a moderate left-sided pleural effusion.

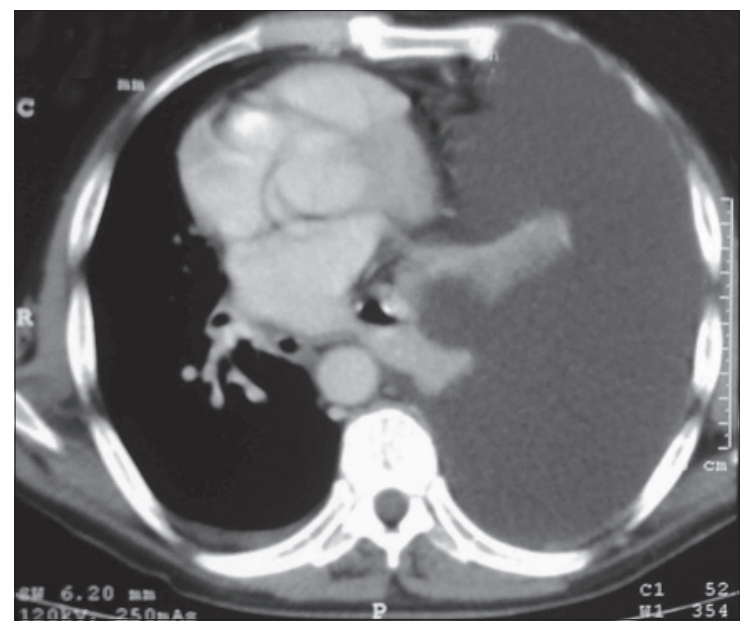

Figure 1: Contrast-enhanced computed tomography showing massive left-sided pleural effusion at the time of admission

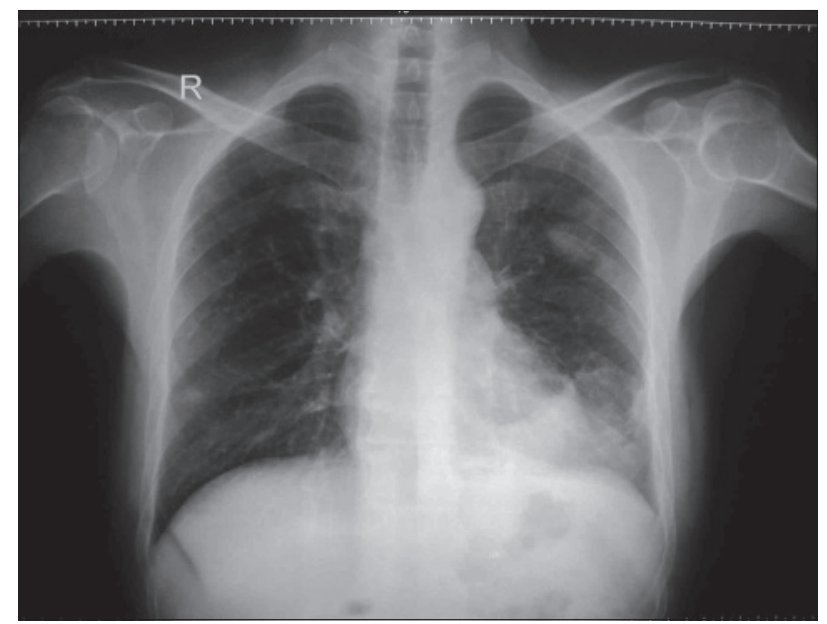

Figure 3: Chest x-ray showing complete resolution of pleural effusion after placement of a stent in the main pancreatic duct
Diagnostic pleurocentesis was performed, which showed protein $5 \mathrm{~g} / \mathrm{dl}$ and amylase $26240 \mathrm{IU} / \mathrm{L}$. A CT scan of the chest and abdomen was done, which showed a moderate left pleural effusion, cholelithiasis, and a pseudocyst in the tail of pancreas, along with ductal changes of pancreatitis [Figure 4]. ERCP was done with the purpose of $\mathrm{PD}$ stenting, A pancreatogram revealed a leak from the tail into the pseudocyst and a $5 \mathrm{~F}$ stent was placed in the pancreatic duct [Figure 5]. A chest $\mathrm{x}$-ray done after three days showed resolution of the pleural effusion [Figure 6]. There was no recurrence of pleural effusion at the two-month follow-up.

\section{Discussion}

Pleural effusion in pancreatic disease occurs due to two mechanisms. The first is reactionary pleural effusion due to pancreatitis, which is usually small and left-sided (may be bilateral). It is characterized by a normal amylase level $(<1000 \mathrm{U} / \mathrm{L})$ and low protein concentration $(<3 \mathrm{~g} / \mathrm{dl}) \cdot{ }^{[4]}$ This type of effusion is seen in acute pancreatitis and resolves

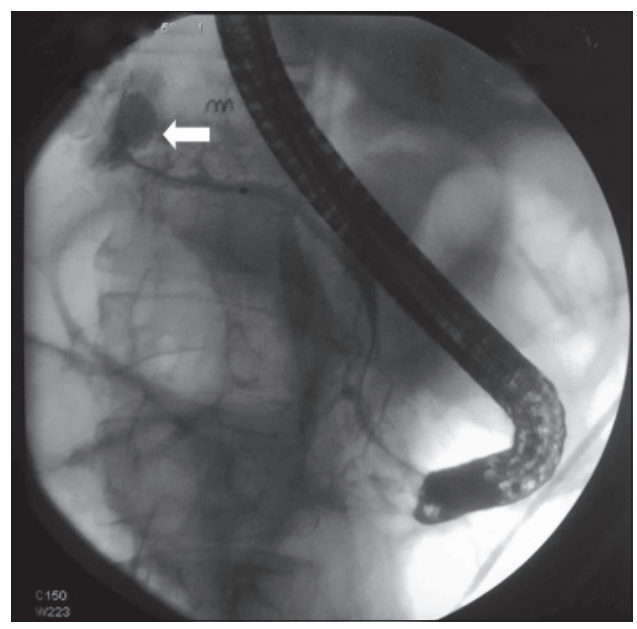

Figure 2: Pancreatogram at ERCP showing dilated main pancreatic duct with leak of contrast from distal body. (Marked with arrow)

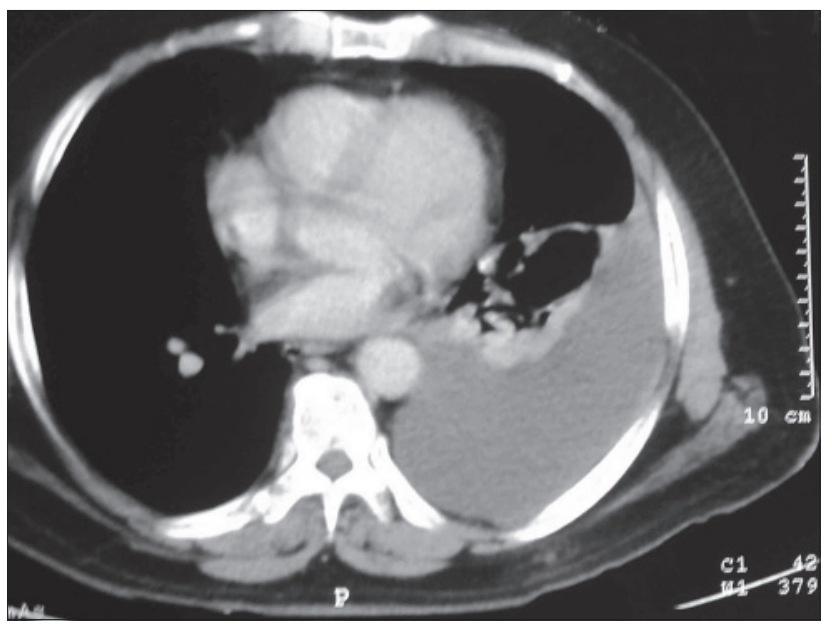

Figure 4: CECT chest showed moderate left pleural effusion at admission 


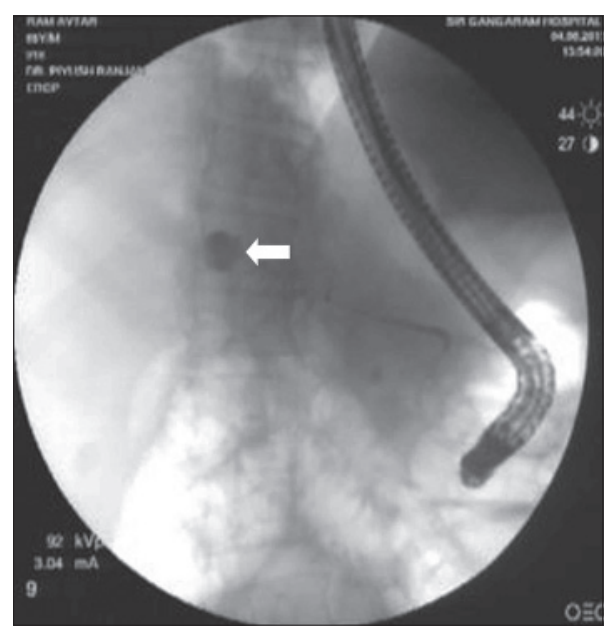

Figure 5: Pancreatogram at ERCP revealing leak from the tail filling the pseudocyst

spontaneously, with recovery of the disease. The second type of pleural effusion in patients with pancreatitis is usually large, left-sided, recurrent, and has a high level of amylase (> $1000 \mathrm{U} / \mathrm{L})$ and proteins (> $3 \mathrm{~g} / \mathrm{dl}$ ). This type of effusion is seen in both chronic and recurrent pancreatitis. In this type of effusion, fluid accumulates in the pleural cavity due to a fistulous communication between either a pancreatic duct or a pseudocyst and the pleura. ${ }^{[5]}$

The underlying mechanism for PPF is usually a leak from the pseudocyst, but a direct pancreatic duct leak also been reported. The fistulous tract passes either through the aortic or esophageal diaphragmatic orifices or directly, transdiaphragmatically. If pancreatic duct disruption develops posteriorly, the pancreatic secretions flow into the retroperitoneum and may dissect into the mediastinum through the aortic or esophageal hiatus and form a pleural fistula. Uncommonly a mediastinal pseudocyst ruptures into the pleural space and forms a PPF. If the disruption occurs anteriorly and is not walled off, a pancreatic-peritoneal fistula develops, which manifests as pancreatic ascites. ${ }^{[6]}$

The clinical features are often variable, but overall, pulmonary symptoms are more common than abdominal symptoms. According to Ali et al., ${ }^{[7]}$ the most common symptoms are due to pleural effusion, that is, dyspnea in $65 \%$, cough in $27 \%$, and chest pain in $23 \%$. Abdominal pain has been reported in $29 \%$ of the cases. Our first case had a typical presentation of PPF, but in the second case, PPF was an incidental finding.

Clinically, a massive pleural effusion with a high fluid amylase level and increased protein concentration in a patient with pancreatitis suggests a diagnosis of PPF. ${ }^{[4,7]}$ Other causes of high amylase in the pleural fluid are, acute pancreatitis, pneumonia, liver cirrhosis, pulmonary tuberculosis, esophageal perforation, female reproductive tract malignancy, lung cancer, metastatic carcinoma, lymphoma, and leukemia.

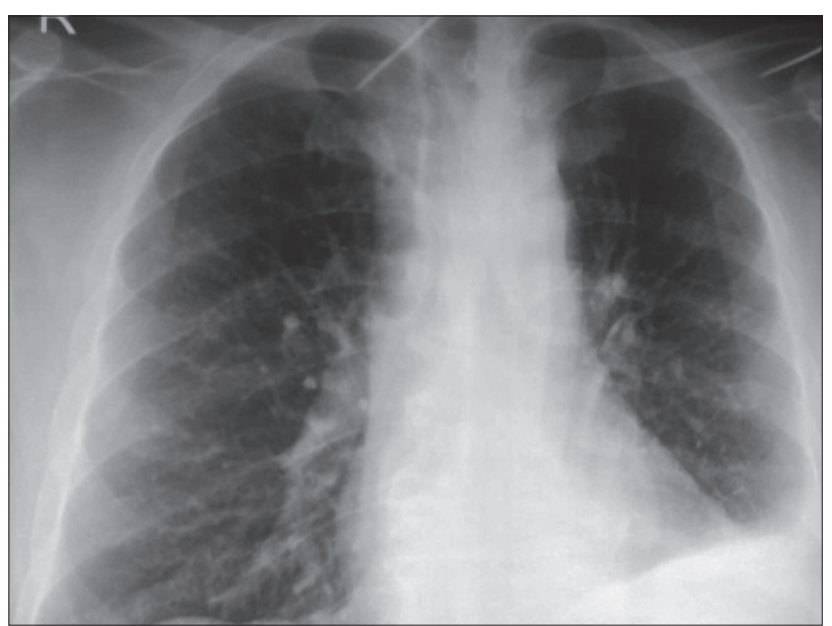

Figure 6: Chest x-ray showing resolution of pleural effusion after placement of main pancreatic duct stent

A direct demonstration of this fistulous communication is difficult. An ultrasound is not a good imaging modality, as the bowel loops interfere with the image quality. ${ }^{[8]}$ A CT scan is very useful in determining the size of the effusion and also reveals changes of the pancreatitis. A CT scan may demonstrate the fistulous tract, especially if obtained immediately after an ERCP. Computed tomography has been done in most studies reporting PPFs, but it is able to demonstrate fistula only in $33-47 \%$ of the cases, because of limitation of resolution of the CT and the poorly enhancing walls of the fistula, which is usually masked within the pseudocysts. ${ }^{[7]}$ Magnetic resonance cholangiopancreatography (MRCP) is considered the investigation of choice for suspected $\mathrm{PPF}$ with a sensitivity of $80 \%$. It provides information about the pancreatic duct beyond the stricture. In addition, a small pseudocyst, peripancreatic collection, and the PPF can be seen. It is useful where ERCP fails, to give adequate information about the ductal anatomy. ${ }^{[9]}$ Multiple pseudocysts or ascites limit the resolution of MRCP. ${ }^{[10]}$ ERCP demonstrates a leak from the main pancreatic duct with a sensitivity of $46-78 \%$. ERCP may not be useful in patients in whom the site of the ductal disruption is distal to the stricture. The main advantage of ERCP is its therapeutic ability apart from imaging. In our cases, diagnosis was possible with a chest x-ray and pleural fluid analysis in both the cases. A CT scan could not demonstrate the fistulous tract and ERCP demonstrated a leak from the main pancreatic duct in both the cases.

Therapeutic options for PPF include medical treatment, endoscopic management, and surgery. The aim of medical treatment is to reduce pancreatic exocrine secretions. Somatostatin analogs are most commonly used along with thoracentesis and/ or tube thoracostomy, which encourage the apposition of pleural surfaces. Medical treatment is usually attempted for two to three weeks. Octreotide administration along with ERCP and stenting has been used for a longer period (2.5 to 6 months). ${ }^{[11]}$ Octreotide is given in an initial dosage of $50 \mathrm{ug}$, administered subcutaneously three times a day, and the dose is titrated based upon the fistula 
output. The maximal dose employed is $250 \mathrm{ug}$, three times daily. ${ }^{[11,12]}$ It is reported that octreotide significantly reduces the fistula output and decreases the time to fistula closure. ${ }^{[12]}$ Measures like the prohibition of oral intake, nasogastric or nasojejunal tube insertion, and total parenteral nutrition used in the past are no longer necessary. The reported success rate of medical management is $30-60 \% .{ }^{[13]}$ However, patients who fail medical treatment have higher rates of complications if they undergo surgery.

Endoscopic retrograde cholangiopancreatography has been used to treat PPF in cases where there is a leak from the main pancreatic duct without a proximal stricture. Therapeutic options at ERCP include endoscopic papillotomy, nasopancreatic tube placement, and placement of a stent in the main pancreatic duct.
Endoscopic papillotomy alone may help in fistula closure by producing a lower pressure gradient for drainage of pancreatic secretions. ${ }^{[11]}$ Insertion of a nasopancreatic tube has been used for one week, followed by placement of an endoprosthesis in the pancreatic duct, if the fistula persists. ${ }^{[6,14]}$ The advantage of a nasopancreatic tube, in contrast to stent placement, is that it allows the pancreaticogram to be obtained repeatedly, without further invasive procedures. It also allows application of low intermittent suction, which may potentially facilitate closure of a leak or fistula. ${ }^{[14]}$ However, the major drawbacks include the necessity for continued hospitalization and patient discomfort due to the presence of the tube in the nose. ${ }^{[14]}$ Stenting decompresses the duct and can bridge the site of ductal disruption. Fistulae, which arise from the head or body, are most suitable for bridging with a stent, however, it

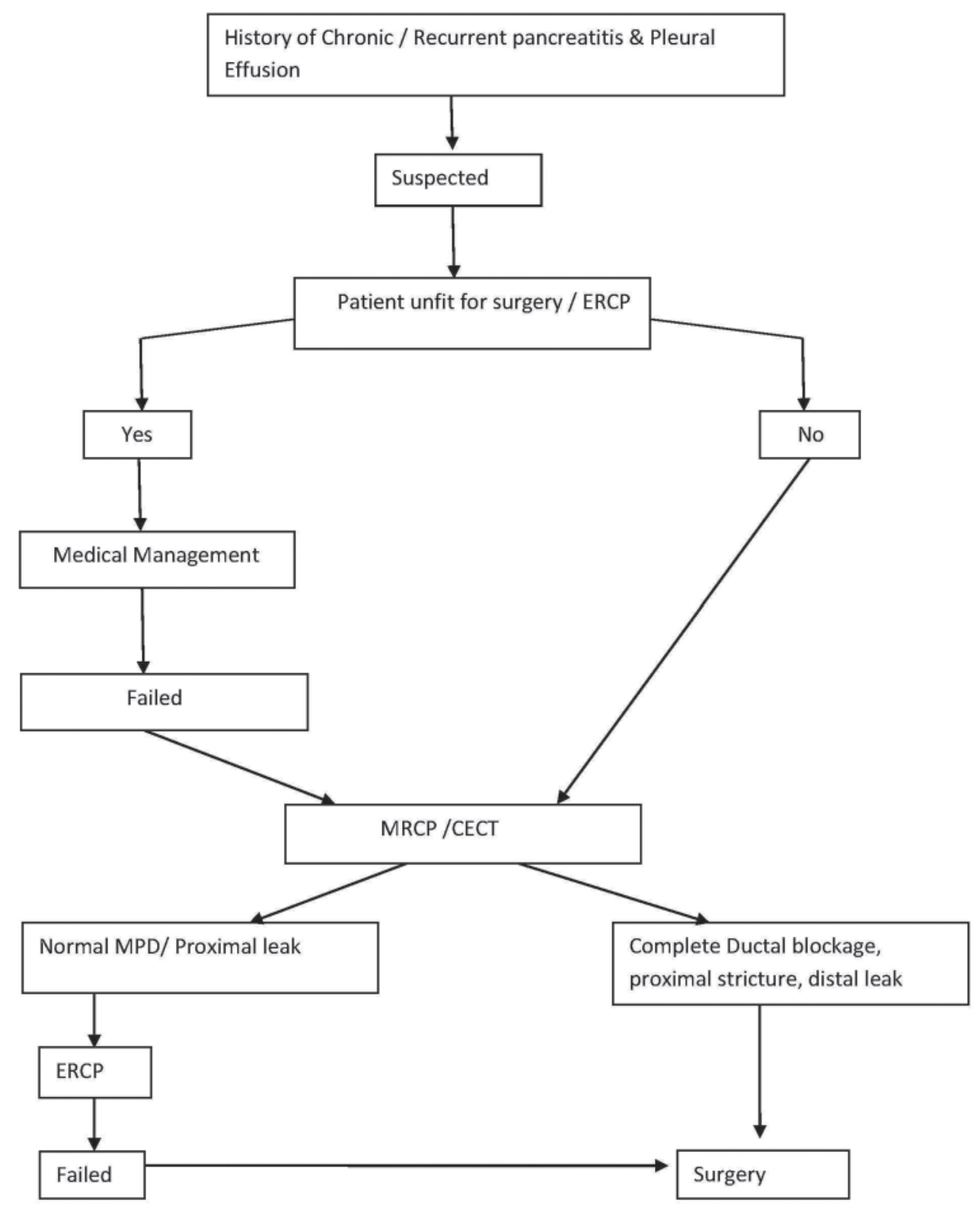

Figure 7: Proposed algorithm for treatment of PPF 
may not be possible when the fistula arises from the tail of the pancreas and the stent may have to be placed close to the duct disruption. ${ }^{[6,14]}$ Bridging pancreatic stents help to close the fistula rapidly by decreasing the ductal pressure and by abolition of the pancreatic pressure gradient. Either a $5 \mathrm{~F}$ or $7 \mathrm{~F}$ size of stents are used in a pancreatic duct. ${ }^{\left[{ }^{6,14]}\right.}$ The optimum duration of drainage for fistulae is unknown, but can vary from four to twelve weeks. In patients with a stricture, the stent may be changed sequentially from two to twelve months ${ }^{[6]}$ Assessment of the persistence of fistula can be done by repeating ERCP at six-week intervals and documenting the leak of the dye. ${ }^{[1]}$ However, long-term use of a stent causes ductal changes that may persist even after its removal. ${ }^{[15]}$ As data on the long-term consequence of stent placement is lacking, it is difficult to draw a definite conclusion. ${ }^{[6]}$ A significant proportion of these patients may require surgery, particularly for persistent, recurrent fluid collections. ${ }^{[16]}$ The issue of how long to continue with endoscopic treatment is still unresolved. ${ }^{[1]}$

In both reported cases, ERCP and stenting of the main pancreatic duct led to the complete resolution of PPF, despite the site of the leak being in the distal PD and the stent not bridging the leak. The probable reason for this was lack of proximal stricture or stone.

Surgery is usually considered in patients who fail conservative and endoscopic treatment. ${ }^{[12,13]}$ Surgical treatment includes either pancreatic resection or enteropancreatic anastomosis to the site of pancreatic duct leakage or to the pseudocyst. ${ }^{[11,12]}$ If there is an obstruction of the main pancreatic duct proximal to the fistula, surgical treatment is necessary to decompress the obstructed duct. Cystogastrostomy, cystojejunostomy, and distal and middle pancreatectomy are appropriate options in the setting of symptomatic pancreatic pseudocysts or pancreatic duct obstruction. ${ }^{[11-13]}$

In a review of 63 patients, King et al., reported that medical therapy was successful only in $31 \%$ of the cases, whereas, surgical therapy was likely to succeed more than three times (94\%), when applied as either an initial strategy or after failed medical management. ${ }^{[13]}$ Complications were noted in $16 \%$ of the patients and death occurred in $3 \%$. It was also noted that $70 \%$ of the complications that followed surgical intervention were seen in patients in whom conversion from medical to operative therapy was required. Most of the patients were initially treated with medical therapy (87\%) and it was deemed to have failed after an average period of $35 \pm 5$ days. The total duration of therapy for patients in whom surgery was required after attempted medical management was $40 \pm 6$ days, which was greater than the 'surgery-alone' cohort. ${ }^{[13]}$ These observations strongly advocate that surgery should be the first line of treatment, if expertise is available.

\section{Conclusion}

Pancreaticopleural fistula should be considered as a diagnosis in cases of pleural effusion, in patients with chronic or recurrent pancreatitis. Pleural fluid amylase estimation is sufficient to establish the diagnosis. CT and/or MRCP are the main imaging modalities to demonstrate PPF. Treatment options are medical, ERCP, and surgery. The treatment algorithm for PPF is not well-defined. We propose a treatment algorithm in patients with PPF, which takes into account the general condition of the patient, the pancreatic ductal anomaly, and the response to treatment [Figure 7].

\section{References}

1. Schoefl R, Haefner M, Pongratz S, Pfeffel F, Stain C, Poetzi R, et al. Endoscopic treatment of fistulas and abscesses in pancreatitis: Three case reports. Endoscopy 1996;28:776-9.

2. Burgess NA, Moore HE, Williams JO, Lewis MH. A review of pancreatico-pleural fistula in pancreatitis and its management. HPB Surg 1992;5:79-86.

3. Olakowski M, Mieczkowska-Palacz H, Olakowska E, Lampe P. Surgical management of pancreaticopleural fistulas. Acta Chir Belg 2009;109:735-40.

4. Branca P, Rodriguez RM, Rogers JT, Ayo DS, Moyers JP, Light RW. Routine measurement of pleural fluid amylase is not indicated. Arch Intern Med 2001;161:228-32.

5. Kaman L, Behera A, Singh R, Katariya RN. Internal pancreatic fistulas with pancreatic ascites and pancreatic pleural effusions: Recognition and management. ANZ J Surg 2001;71:221-5.

6. Safadi BY, Marks JM. Pancreatic-pleural fistula: The role of ERCP in diagnosis and treatment. Gastrointest Endosc 2000;51:213-5.

7. Ali T, Srinivasan N, Le V, Chimpiri AR, Tierney WM. Pancreaticopleural fistula. Pancreas 2009;38:e26-31.

8. Ferri E, Pisani M. Pleuro-pancreatic fistula diagnosed by ultrasonography. Chir Ital 2002;54:397-400.

9. Vyas S, Gogoi D, Sinha SK, Singh P, Yadav TD, Khandelwal N. Pancreaticopleural fistula: An unusual complication of pancreatitis diagnosed with magnetic resonance cholangiopancreatography. JOP 2009;10:671-3.

10. Tajima Y, Tsutsumi R, Kuroki T, Mishima T, Adachi T, Kitasato A, et al. Evaluation and management of thoracopancreatic fistula. Surgery 2006;140:773-8.

11. Dhebri AR, Ferran N. Nonsurgical management of pancreaticopleural fistula. JOP 2005;6:152-61.

12. Rockey DC, Cello JP. Pancreaticopleural fistula. Report of 7 patients and review of the literature. Medicine (Baltimore) 1990;69:332-44.

13. King JC, Reber HA, Shiraga S, Hines OJ. Pancreatic-pleural fistula is best managed by early operative intervention. Surgery 2010;147:154-9.

14. Neher JR, Brady PG, Pinkas H, Ramos M. Pancreaticopleural fistula in chronic pancreatitis: Resolution with endoscopic therapy. Gastrointest Endosc 2000;52:416-8.

15. Griesshammer B, Strobel M. Pancreaticopleural fistula treated by transpapillary implantation of a plastic prosthesis. Endoscopy 1998;30:741.

16. Pottmeyer EW $3^{\text {rd }}$, Frey CF, Matsuno S. Pancreaticopleural fistulas. Arch Surg 1987;122:648-54.

How to cite this article: Ranjan P, Bansal R, Sachdeva M, Kumar M. Pancreaticopleural fistula: Report of two cases and review of literature. J Dig Endosc 2013;4:123-7.

Source of Support: Nil, Conflict of Interest: None declared. 